\title{
Direct observation of atomic diffusion in warm rubidium ensembles
}

\author{
Michał Parniak $\cdot$ Wojciech Wasilewski
}

Received: 12 August 2013/ Accepted: 20 October 2013/Published online: 16 November 2013

(C) The Author(s) 2013. This article is published with open access at Springerlink.com

\begin{abstract}
We present a robust method for measuring diffusion coefficients of warm atoms in buffer gases. Using optical pumping, we manipulate the atomic spin in a thin cylinder inside the cell. Then, we observe the spatial spread of optically pumped atoms in time using a camera, which allows us to determine the diffusion coefficient. As an example, we demonstrate measurements of diffusion coefficients of rubidium in neon, krypton and xenon acting as buffer gases. We have determined the normalized (273 $\mathrm{K}, 760$ Torr) diffusion coefficients to be $0.18 \pm 0.03 \mathrm{~cm}^{2} / \mathrm{s}$ for neon, $0.07 \pm 0.01 \mathrm{~cm}^{2} / \mathrm{s}$ for krypton and $0.052 \pm 0.006$ $\mathrm{cm}^{2} / \mathrm{s}$ for xenon.
\end{abstract}

\section{Introduction}

Warm atomic ensembles have recently become a very useful tool in modern quantum engineering. The most notable applications include quantum memories $[1,2]$ and quantum repeaters [3] that can lead to the development of quantum networks [4]. Warm atoms have also been used as a medium for four-wave mixing [5], electromagnetically induced transparency (EIT) [6] and ultraprecise magnetometry [7].

While experiments with vapors contained in sealed cells are relatively simple, they are limited by inevitable atomic motion. Typically, a buffer gas is used to contain the atoms and make their motion diffusive. In all of the above

\footnotetext{
M. Parniak ( $₫) \cdot$ W. Wasilewski $(\bowtie)$

Institute of Experimental Physics, University of Warsaw, Hoża 69, 00-681 Warsaw, Poland

e-mail: michal.parniak@student.uw.edu.pl

W. Wasilewski

e-mail: wwasil@fuw.edu.pl
}

examples, the diffusion rate was among primary performance-limiting factors. Its importance has been recognized and its effect on EIT [8-11] and on the gradient echo memory [12-14] has been studied both experimentally and theoretically.

Prior knowledge of diffusion coefficients enables designing optimal experiments and greatly facilitates the interpretation of the results. However, there is a striking lack of precise measurements of diffusion coefficients in various buffer gases. We believe that the reason for this is unavailability of robust methods. In most cases, diffusion coefficients were deduced using methods designed for studying spin exchange of optically aligned atoms [15].

The lack of both data and methods motivated us to develop a simple and robust procedure designed specifically to measure the diffusion coefficients. We demonstrate it on an example of diffusion of rubidium in neon, krypton and xenon.

In our method, we pump optically a thin pencil-shaped volume of atoms inside a given cell using a short laser pulse. After the pump pulse ends, we wait for a varying time and let rubidium atoms diffuse. Then, we send a pulse from a probe laser in a beam that covers nearly the entire cell. The probe light is virtually unaffected by pumped atoms but absorbed by the unpumped ones. Therefore, spatial distribution of the transmitted probe light reveals how far the atoms have travelled between the pump and probe pulses and thus provides the diffusion coefficient.

This paper is organized as follows. In Sect. 2, we present a simple model that describes our method. In Sect. 3, we describe in detail our experimental setup. In Sect. 4, we present practical methods for analyzing the data obtained. Section 5 gives the results of our measurements and compares them with theoretical predictions as well as with 
the results obtained previously. Section 6 concludes the paper.

\section{Method}

In our method, we register a decrease in optical depth of the atomic sample due to optical pumping $\Delta \mathrm{OD}(x, y, t)$ as a function of spatial position $(x, y)$ and the delay $t$ between the pumping and the actual observation. In practice, the difference $\Delta \mathrm{OD}$ may be computed by measuring the intensity of light that passes through our cell with $I_{\mathrm{p}}(x, y, t)$ and without optical pumping $I_{\mathrm{np}}(x, y)$. The formula reads:

$\Delta \mathrm{OD}(x, y, t)=\ln \left(\frac{I_{\mathrm{p}}(x, y, t)}{I_{\mathrm{np}}(x, y)}\right)$.

The decrease in optical depth is also proportional to the decrease in density of the atoms in the ground state of the atomic transition to which the probe is coupled, that is, $c_{\mathrm{np}}-c_{\mathrm{p}}(x, y, t)$, where $c_{\mathrm{np}}$ stands for the equilibrium density observed without pumping. Since the density $c_{\mathrm{p}}(x, y, t)$ at time $t$ after pump pulse evolves according to the diffusion equation, so does the decrease in the optical depth $\Delta \mathrm{OD}(x, y, t)$.

In addition to the diffusion, other mechanisms, such as spin-exchange collisions, may urge the density $c_{\mathrm{p}}(x, y, t)$ toward the steady-state value $c_{\mathrm{np}}$. We call the rate of those relaxation processes $\gamma_{0}$ and observe that it is position independent. Later, we incorporate it into data analysis. Moreover, the atoms may relax in collisions with cell walls. In our experiments, the observation times $t$ were too short for pumped atoms to reach the side walls. Some portion of the initially pumped volume would reach cell windows; however, since the length of the cell is almost 100 times the typical diffusion distance, the boundary effects are negligible.

It is convenient and intuitive to assume that the density $c_{\mathrm{p}}(x, y, t)$ after pumping is $z$-independent, which requires that the pump beam should saturate the absorption in the ensemble. In fact, this assumption is not necessary, as every solution to the $3 \mathrm{D}$ diffusion equation (in this case with additional relaxation) can be written as sum of separable solutions, that is, $c_{\mathrm{np}}-c_{\mathrm{p}}(x, y, z, t)=\sum_{j}$ $u_{j}(x, y, t) f_{j}(z, t) \quad \exp \left(-\gamma_{0} t\right)$, where both $u_{j}(x, y, t)$ and $f_{j}(z, t)$ satisfy the diffusion equation in respective coordinates. Now, we integrate over $z$ to finally obtain the decrease in the optical depth $\Delta \operatorname{OD}(x, y, t)$, but the integral $\int_{0}^{L} f_{j}(z, t) \mathrm{d} z$ is time independent, as the relaxation at optical windows is negligible. The optical depth difference will satisfy the 2D diffusion equation with relaxation, as it is now a linear combination of $u_{j}(x, y, t)$ with time-independent coefficients.
By fitting Gaussians to obtained $\Delta \mathrm{OD}(x, y, t)$ crosssections as presented in Fig. 1, we can estimate the diffusion coefficient $D$ using the diffusion rule for the width of fitted Gaussians $\sigma_{x}(t)=\sqrt{\sigma_{x}(t=0)^{2}+4 D t}$. A more universal method is presented in Data Analysis section.

\section{Experimental setup}

Figure 2 presents a schematic of the experimental setup. Both pump and probe lasers are Toptica distributed feedback laser diodes, each frequency stabilized using dichroic atomic vapor spectroscopy [16].

In case of rubidium 87, the pump laser was tuned to $F_{g}=1 \rightarrow F_{e}=0,1,2$ transitions on D2 line and the probe laser to $F_{g}=1 \rightarrow F_{e}=2$ transition on D1 line. In case of rubidium 85 , the pump laser was tuned to $F_{g}=3 \rightarrow$ $F_{e}=2,3,4$ transitions on D2 line and the probe laser to $F_{g}=3 \rightarrow F_{e}=2,3$ transitions on D1 line. We have found that the stability of the pump laser is important, as the pumping rate needs to be constant, although the laser does not need to be tuned precisely to the center of any transition.

The laser beams are directed onto the acousto-optic modulators (AOM) used to produce the pulse sequence. The beam shaping optics follows. Both beams are collimated, and the probe beam is expanded to a desired $1 / e^{2}$ waist diameter of about $1 \mathrm{~cm}$, while the pump beam has a waist diameter of $1 \mathrm{~mm}$.

The beams are joined on a polarizing beam splitter (PBS). Half-wave plates before the joining point allow us to control the power of both beams precisely. After the PBS, the beams are parallel and overlap.

Both beams pass through a quartz cell (Precision Glassblowing, $25 \mathrm{~mm}$ in diameter, various lengths) filled

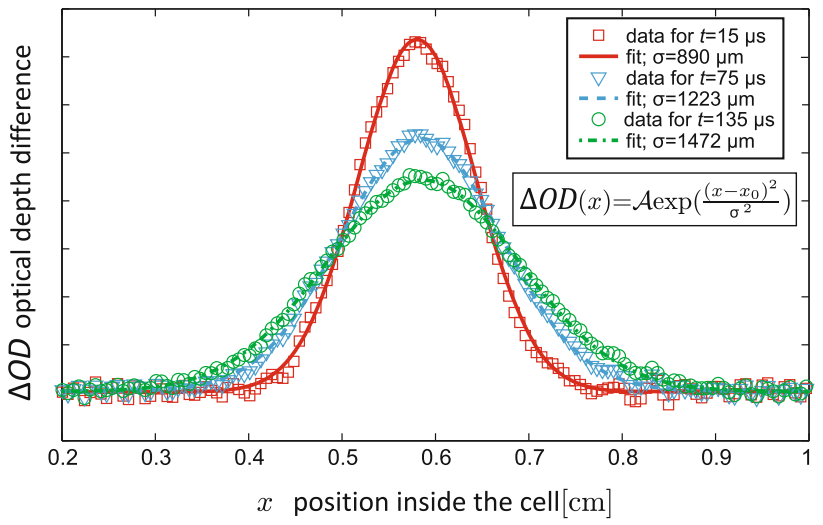

Fig. 1 Typical examples of obtained optical depth difference $\Delta \mathrm{OD}(x, y=0, t)$ maps at various times $t$ after pump pulse. Gaussian fits give us widths of these distributions $\sigma_{x}(t)$ as a function of diffusion time 
Fig. 2 Schematic of our experimental setup. AOM acousto-optic modulator, $D A V L L$ dichroic atomic vapor laser lock setup [16], $P D$ photodiode, $P B S$ polarizing beam splitter, $R b$ rubidium vapor cell, $\lambda / 2$ half-wave plate, $\lambda / 4$ quarter-wave plate, $I F$ interference filter, $C C D$ chargecoupled device camera. Lenses are labeled with their focal lengths

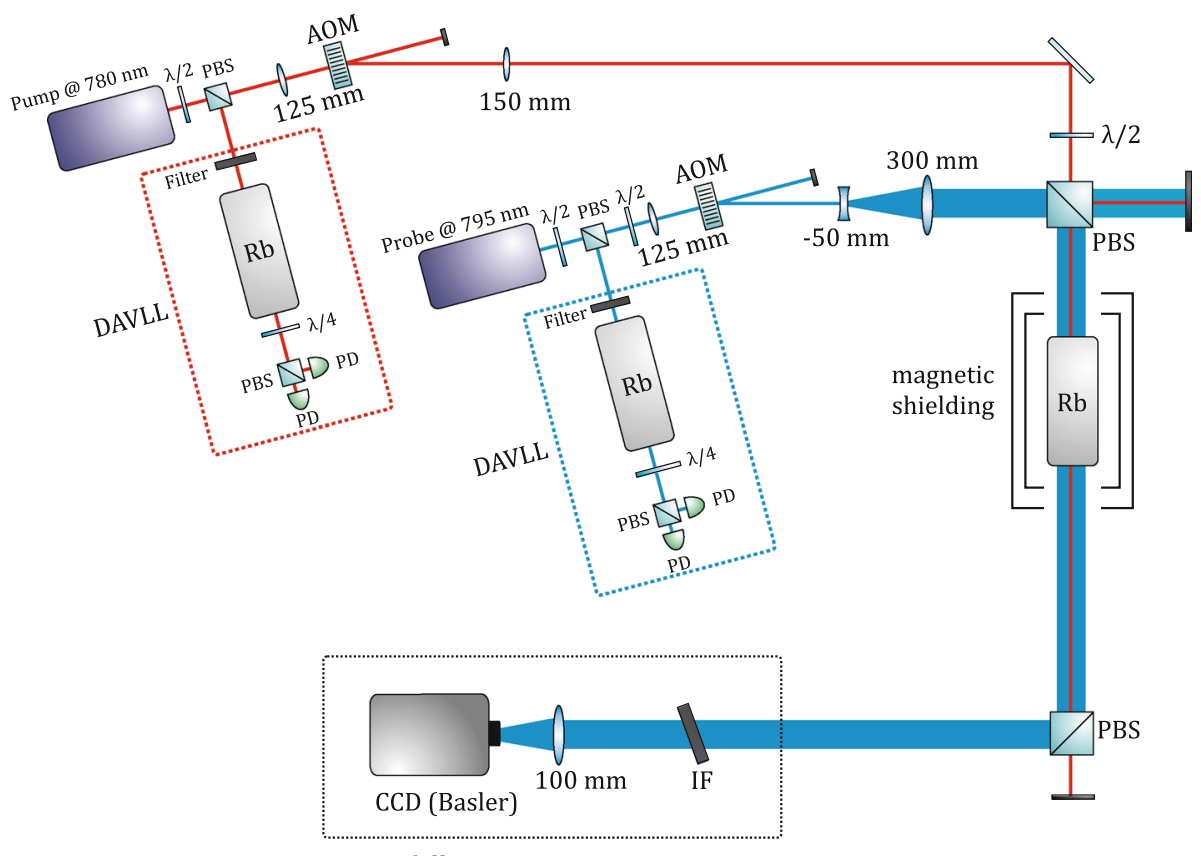

diffusion measurement

with isotopically pure rubidium vapor and buffer gas. The vapor cell is placed inside a $\mu$-metal magnetic shielding to avoid pumping alternations due to external magnetic filed. The cell is mounted in flexible aluminum sleeves heated with a bifilar-wound copper coil. Despite the bifilar winding, we have found that it is better to stop the heating for the time of measurement to avoid disturbing the pumping. The holder temperature stabilization is based on the resistance of the coil's windings.

The vapor temperature is determined by measuring absorption spectrum of the cell and fitting the result with a theoretical curve. We keep the temperature within the $5{ }^{\circ} \mathrm{C}$ range around $40{ }^{\circ} \mathrm{C}$.

After passing through the cell, the pump beam is filtered out by a PBS and an interference filter. We image the inside of the cell on a CCD camera (Basler, scA1400$17 \mathrm{fm}$ ) with a single lens. Magnification of this optical system was both calculated and measured with a cell replaced by a reference target. These two methods led to consistent results, showing that camera's pixel pitch corresponds to a $59 \pm 2 \mu \mathrm{m}$ distance inside the cell.

The camera is triggered synchronously with laser pulses. We use minimal shutter duration (40 $\mu$ s) to minimize the background coming mainly from scattering on the AOMs. In addition, by controlling the time when shutter opens, we minimize the amount of pump light registered by the camera. We achieved a relatively low background level, dominated by the electronic offset. It was sufficient to subtract constant background intensity from each image frame.

The pulse sequence is represented schematically in Fig. 3. To measure the reference intensity of the probe light

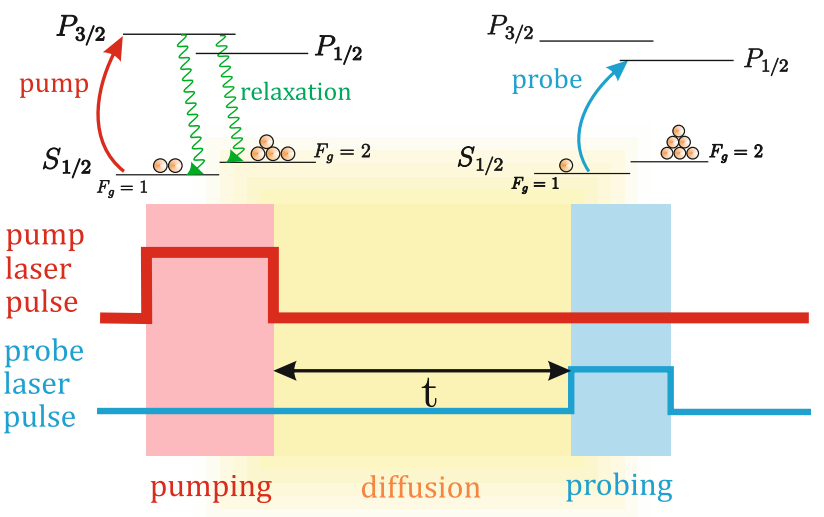

Fig. 3 Light pulse sequence we use in our experiment. The top line represents the pump laser tuned to rubidium D2 line, and the bottom line represents the probe laser tuned to D1 line. On the energy level diagrams (in this case of rubidium 87 atom), we show how the optical pumping influences populations: during pumping on the left and during probing on the right. Wavy arrows represent relaxation mainly due to spontaneous emission

$I_{\mathrm{np}}(x, y)$, we send a $0.4 \mu$ s probe pulse alone and image the beam shape after passing through the cell. To measure the probe light intensity with the optical pumping present $I_{\mathrm{p}}(x, y, t)$, first we apply a $0.5 \mu$ s pump pulse and then wait for a varying time $t$ and apply a $0.4 \mu$ s probe pulse. The initial $\Delta \mathrm{OD}(x, y, t=0)$ is diffused only slightly due to the short pump pulse. The unsaturated absorption of the pump light was about $70 \%$, but saturation effects caused half of the pump pulse to be transmitted through the cell. It resulted in optical pumping being weakly dependent on $z$. The delay time $t$ varies from 1 to $150 \mu$ s. Each collected 
image of the probe beam is averaged over 50 measurements, and the entire collection sequence for all delays $t$ is repeated 10 times. This allows us to average over both long-term ( $\mathrm{min})$ and short-term (ms) fluctuations. The data collection rate is limited by the $40 \mathrm{~Hz}$ frame rate of the camera.

\section{Data analysis}

The initial shape of the pumped region is not easily described analytically; therefore, we have elected to use a data analysis method that can deal with arbitrary functions. It is based on solving the diffusion equation in the spatial frequency Fourier domain, which is more general than a Gaussian fit from Fig. 1. A similar method has previously been used in studies of fluorescence redistribution after photobleaching [17]. Let us define the spatial Fourier transform of the decrease in optical depths of the atomic sample due to optical pumping $\Delta \mathrm{OD}(x, y, t)$ as:

$$
\begin{aligned}
\mathcal{F}\{\Delta \mathrm{OD}\}\left(k_{x}, k_{y}, t\right)= & \frac{1}{2 \pi} \iint \mathrm{d} x \mathrm{~d} y \Delta \mathrm{OD}(x, y, t) \exp \left(-i k_{x} x\right. \\
& \left.-i k_{y} y\right) .
\end{aligned}
$$

As we discussed in Sect. 2, the $z$-dependence of atomic density can be separated and $\Delta \mathrm{OD}(x, y, t)$ becomes a linear combination of functions satisfying $2 \mathrm{D}$ diffusion equation with relaxation, so the time evolution of this Fourier transform due to diffusion takes on a simple form

$$
\begin{aligned}
& \mathcal{F}\{\Delta \mathrm{OD}\}\left(k_{x}, k_{y}, t\right) \\
& \left.=\mathcal{F}\{\Delta \mathrm{OD}\}\left(k_{x}, k_{y}, 0\right) \exp \left(-(\underbrace{\gamma_{0}+D\left(k_{x}^{2}+k_{y}^{2}\right.}_{\gamma(\mathbf{k})})\right) t\right) .
\end{aligned}
$$

This solution tells us that a component of optical depth difference having a certain spatial periodicity given by $k_{x}$ and $k_{y}$ (that may be also called spatial frequencies) decays exponentially at a rate

$\gamma\left(k_{x}, k_{y}\right)=\gamma_{0}+D\left(k_{x}^{2}+k_{y}^{2}\right)$.

The procedure of data analysis according to the above equations is as follows. Having measured optical depth differences $\Delta \mathrm{OD}(x, y, t)$ for various delay times $t$, we perform a numerical Fourier transform of each map and get $\mathcal{F}\{\Delta \mathrm{OD}\}\left(k_{x}, k_{y}, t\right)$ for different delay times $t$.

Next, for each $\mathbf{k}=\left(k_{x}, k_{y}\right)$, we fit a simple exponential decay model to the absolute amplitude of a component of the optical depth decrease with a given spatial periodicity. In practice, this is possible only for components of high enough amplitudes, that is, for $|\mathbf{k}|$ smaller than the inverse pump beam width. The fit result is the decay rate as a function of spatial periodicity $\gamma(\mathbf{k})$. Exemplary decay fits for various spatial wave vectors $\mathbf{k}$ are presented in Fig. 4.
As this decay rate should only depend on the length of $\mathbf{k}$ vector, we perform an angular averaging procedure to obtain a one-variable function $\gamma(|\mathbf{k}|)$. Finally, we use the relation for the decay rate $\gamma(|\mathbf{k}|)=\gamma_{0}+D|\mathbf{k}|^{2}$ that comes from the diffusion equation. We fit a parabola to the previously computed $\gamma(|\mathbf{k}|)$ dependence and retrieve the diffusion coefficient $D$. Exemplary fits for various $\mathrm{Rb}$ cells we used are presented in Fig. 5.

\section{Results}

The presented results were obtained using the Fourier method described above. Special care was taken to ensure

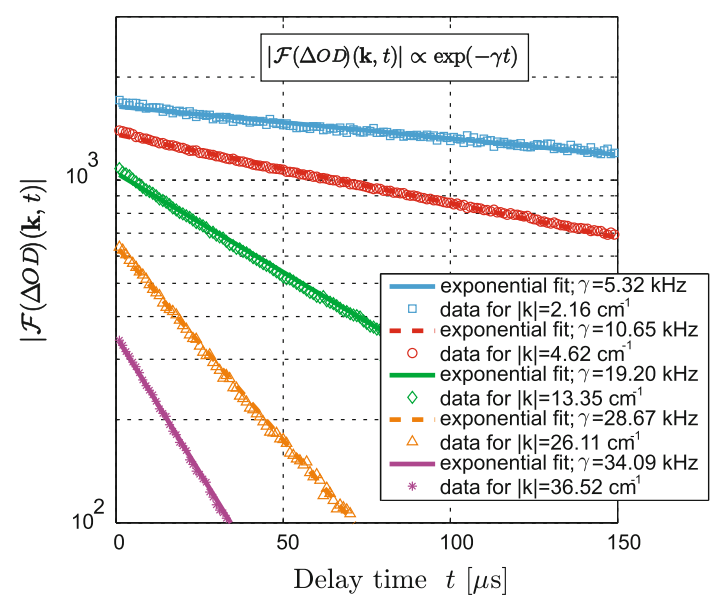

Fig. 4 Exemplary exponential decays of $\mathcal{F}\{\Delta \mathrm{OD}\}(|\mathbf{k}|, t)$ with time between pump and probe, for various components with spatial wave vectors $\mathbf{k}$ in the image of optical depth decrease $\Delta \mathrm{OD}(x, y, t)$. Data for ${ }^{85} \mathrm{Rb}$ cell with 5 Torr of neon

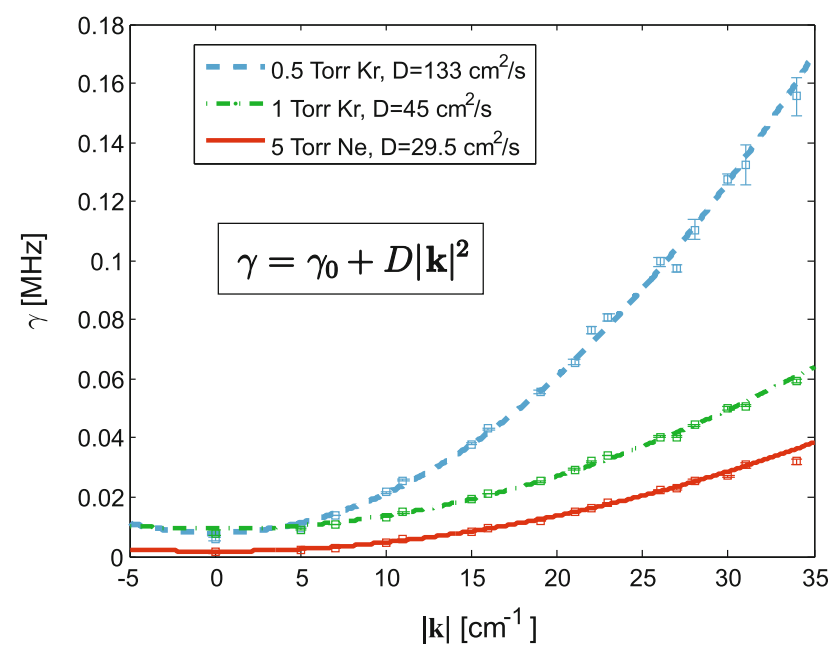

Fig. 5 Quadratic fits of the decay rate $\gamma(|\mathbf{k}|)$ as a function of spatial wave vector $\mathbf{k}$ to the data obtained in the Fourier procedure of data analysis, as shown in Fig. 4 
Table 1 Measured diffusion coefficients, normalized diffusion coefficients obtained from our measurements, theoretical predictions based on the Chapman-Enskog formula and some previous results of the diffusion coefficients measurements

\begin{tabular}{|c|c|c|c|c|c|}
\hline Cell (buffer gas, $\mathrm{Rb}$ isotope) & $\begin{array}{l}D\left(\mathrm{~cm}^{2} / \mathrm{s}\right) \\
\left(40^{\circ} \mathrm{C}\right)\end{array}$ & $\begin{array}{l}D \circ(\text { this paper }) \\
\left(\mathrm{cm}^{2} / \mathrm{s}\right)\end{array}$ & $\begin{array}{l}D \circ(\text { theory }) \\
\left(\mathrm{cm}^{2} / \mathrm{s}\right)\end{array}$ & $\begin{array}{l}D \circ(\text { theory) } \\
\left(\mathrm{cm}^{2} / \mathrm{s}\right)\end{array}$ & $D \circ($ previous results $)\left(\mathrm{cm}^{2} / \mathrm{s}\right)$ \\
\hline Ne 5 Torr, ${ }^{85} \mathrm{Rb}$ & $29.5 \pm 1.0$ & $0.18 \pm 0.02$ & \multirow[t]{4}{*}{$0.18 \pm 0.03$} & \multirow[t]{4}{*}{$0.145 \pm 0.01$} & \multirow{4}{*}{$\begin{array}{c}0.2[18], 0.11[8], 0.18[19] \\
0.31[15,20], 0.48[21]\end{array}$} \\
\hline Ne 2 Torr, ${ }^{87} \mathrm{Rb}$, paraf. & $62.0 \pm 0.8$ & $0.15 \pm 0.02$ & & & \\
\hline Ne 100 Torr, ${ }^{85} \mathrm{Rb}$ & $1.69 \pm 0.04$ & $0.21 \pm 0.03$ & & & \\
\hline Ne 50 Torr, ${ }^{87} \mathrm{Rb}$ & $2.70 \pm 0.04$ & $0.17 \pm 0.02$ & & & \\
\hline Kr 1 Torr, ${ }^{87} \mathrm{Rb}$ & $71 \pm 2$ & $0.08 \pm 0.01$ & \multirow[t]{4}{*}{$0.07 \pm 0.01$} & \multirow[t]{3}{*}{$0.065 \pm 0.005$} & \multirow[t]{3}{*}{$0.068[18], 0.1[22], 0.04[13$} \\
\hline Kr 1 Torr, ${ }^{87} \mathrm{Rb}$, paraf. & $45 \pm 2$ & $0.06 \pm 0.01$ & & & \\
\hline Kr 0.5 Torr, ${ }^{87} \mathrm{Rb}$ & $133 \pm 4$ & $0.08 \pm 0.01$ & & & \\
\hline Xe 1 Torr, ${ }^{87} \mathrm{Rb}$, paraf. & $43 \pm 1$ & $0.052 \pm 0.006$ & & $0.055 \pm 0.005$ & $0.057[18]$ \\
\hline
\end{tabular}

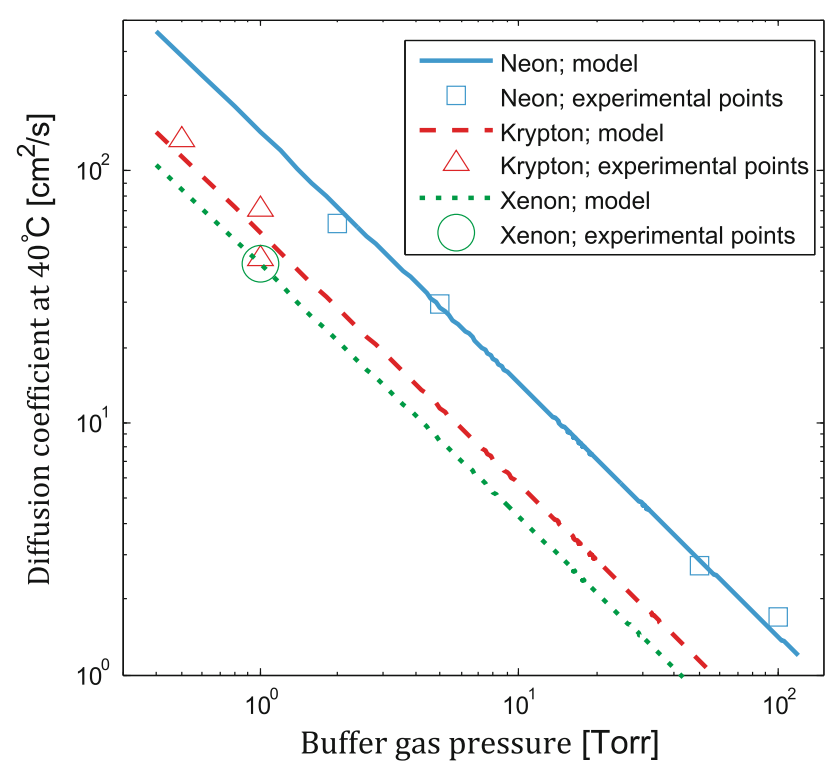

Fig. 6 Summary of obtained diffusion coefficients of rubidium in various buffer gases compared with inverse relation to buffer gas pressure predicted by the Chapman-Enskog model

validity of each step, including verification of rotational symmetry of $\gamma(\mathbf{k})$, which allowed us to proceed with angular averaging to obtain $\gamma(|\mathbf{k}|)$. We noticed that good alignment was critical when ensuring that the spread of values of $\gamma(\mathbf{k})$ for a given $|\mathbf{k}|$ was small. Optimizing the setup resulted in this spread being much smaller than the uncertainty coming from each exponential fit, but both error sources were taken into account when calculating uncertainties.

Table 1 ( $D$, second column) contains diffusion coefficients at $40{ }^{\circ} \mathrm{C}$ of rubidium in various cells, containing neon, krypton or xenon. In Fig. 6, we see that the diffusion coefficient scales with inverse of vapor gas pressure as expected [23] over a broad range of buffer gas pressures, especially for neon. In this figure, we also include curves representing model behavior. The diffusion coefficients we measured in two cells with 1 Torr krypton differ much more than expected. We believe that this could be due to a different true quantity of gas contained in those cells due to variations in the seal-off process. Most likely, the pressure was measured at significantly different temperatures of the cell bodies. Another reason could be significant evaporation of the paraffin coating, which was present only in one cell as chemicals such as volatile hydrocarbons present in the coating could slow down the diffusion when in gaseous state.

To our knowledge, our group has been the first one to measure directly the diffusion coefficient of rubidium in xenon. The result we present here confirms the previously measured value [18].

We scaled the results to normal conditions (760 Torr pressure and $0{ }^{\circ} \mathrm{C}$ temperature) for $\mathrm{Ne}, \mathrm{Kr}$ and $\mathrm{Xe}$ using Chapman-Enskog formula [23]. We inferred uncertainties of normalized diffusion coefficient for neon and krypton from the spread of experimental results. In case of xenon, we attribute the uncertainty of the result to the uncertainty of buffer gas pressure. Note that unnormalized diffusion coefficient for each cell is measured much more accurately. Values of collision integrals at different temperatures were obtained according to the procedure described in Ref. [23]. Required collisional parameters were taken from Refs. [24] and [25]. The ideal gas model was used for the relation between pressure of the buffer gas and temperature. We believe that using the Chapman-Enskog formula and normalized results we present here, it is possible to calculate the diffusion coefficient of rubidium in $\mathrm{Ne}, \mathrm{Kr}$ or $\mathrm{Xe}$ at an arbitrary pressure or temperature.

In 1, we compare our normalized experimental results ( $D$ 。, third column) with theoretical predictions based on Chapman-Enskog formula and studies of atomic collisions ( $D \circ$, fourth column). Our results display satisfactory conformity with theoretical predictions. Note that both the normalization we perform and the theoretical predictions are based on a fundamentally approximate theory, as the 
Chapman-Enskog formula comes from an approximate solution of the Boltzmann equation and requires various parameters that were calculated indirectly.

The results we present here differ significantly from some of the previous results (last column in Table 1); however, we believe ours are more reliable for several reasons. Firstly, the results of this paper agree with the results we obtained using the same cells [18] but a completely different method. Secondly, the former methods relied on measuring the relaxation of the atomic spin alignments, a function of gas pressure to retrieve both the diffusion coefficient and the spin-exchange rates [15].

\section{Conclusions}

We have presented a robust and simple method for measuring the diffusion coefficients of warm atoms in buffer gases. Our method might be used in all systems where the phenomenon of optical pumping occurs. We have shown that observing the spread of an optically pumped region by applying a pulsed probe beam and imaging it on a camera is sufficient to determine the diffusion coefficient of the system. The data analysis involved Fourier transforming of measured optical depth differences and finding decay rates for components of different spatial periodicity. This approach is robust and provides opportunities for data consistency checks at various calculation stages.

Our method can be easily used to measure diffusion in countless physical systems and is easy to implement. In principle, one laser could be sufficient for both pump and probe pulses.

As a demonstration, we have measured the diffusion coefficients of rubidium in neon, krypton and xenon. Our results are consistent with both theoretical predictions and previous results. We have also found the presented method very useful when it comes to characterizing various sealed cells with rubidium and a buffer gas. Notably, it is capable of capturing wide range of diffusion coefficients, of at least two orders of magnitude.

We believe that our measurements will enable greater control and better design of experiments with warm rubidium ensembles. In particular, we note that krypton and xenon appear to be very good buffer gases for modern quantum applications; yet, they have scarcely been used so far. Apart from us, only one group has utilized krypton [26], while xenon has been used only by us [18].

To our knowledge, our group has been the first one to measure actually the diffusion coefficient of rubidium in xenon, with this paper confirming Ref. [18]. Given the recent applications of hyperpolarized ${ }^{129} \mathrm{Xe}$ in medical imaging, we hope that the precise value of this diffusion coefficient will help optimize setups like the one presented in Ref. [27].
Acknowledgments We acknowledge generous support from Konrad Banaszek and Rafał Demkowicz-Dobrzański. This work was supported by the Foundation for Polish Science TEAM project, EU European Regional Development Fund and FP7 FET project Q-ESSENCE (Contract No. 248095), National Science Center Grant No. DEC-2011/03/D/ST2/01941 and Polish NCBiR under the ERANET CHIST-ERA project QUASAR.

Open Access This article is distributed under the terms of the Creative Commons Attribution License which permits any use, distribution, and reproduction in any medium, provided the original author(s) and the source are credited.

\section{References}

1. C.H. van der Wal, M.D. Eisaman, A. André, R.L. Walsworth, D.F. Phillips, A.S. Zibrov, M.D. Lukin, Science 301, 196 (2003). doi:10.1126/science.1085946

2. J. Appel, E. Figueroa, D. Korystov, M. Lobino, A. Lvovsky, Phys. Rev. Lett. 100, 093602 (2008). doi:10.1103/PhysRevLett. 100.093602

3. L.M. Duan, M.D. Lukin, J.I. Cirac, P. Zoller, Nature 414, 413 (2001). doi: $10.1038 / 35106500$

4. H. Kimble, Nature 453, 1023 (2008). doi:10.1038/nature 07127

5. C. McCormick, A. Marino, V. Boyer, P. Lett, Phys. Rev. A 78, 043816 (2008). doi:10.1103/PhysRevA.78.043816

6. M. Fleischhauer, A. Imamoglu, J. Marangos, Rev. Mod. Phys. 77, 633 (2005). doi:10.1103/RevModPhys.77.633

7. W. Chalupczak, R.M. Godun, S. Pustelny, W. Gawlik, Appl. Phys. Lett. 100, 242401 (2012). doi:10.1063/1.4729016

8. M. Shuker, O. Firstenberg, R. Pugatch, A. Ron, N. Davidson, Phys. Rev. Lett. 100, 223601 (2008). doi:10.1103/PhysRevLett. 100.223601

9. O. Firstenberg, M. Shuker, R. Pugatch, D. Fredkin, N. Davidson, A. Ron, Phys. Rev. A 77, 043830 (2008). doi:10.1103/PhysRevA. 77.043830

10. O. Firstenberg, P. London, D. Yankelev, R. Pugatch, M. Shuker, N. Davidson, Phys. Rev. Lett. 105, 183602 (2010). doi:10.1103/ PhysRevLett.105.183602

11. D. Yankelev, O. Firstenberg, M. Shuker, N. Davidson, Opt. Lett. 38, 1203 (2013). doi:10.1364/OL.38.001203

12. Q. Glorieux, J.B. Clark, A.M. Marino, Z. Zhou, P.D. Lett, Opt. Express 20, 12350 (2012). doi:10.1364/OE.20.012350

13. D.B. Higginbottom, B.M. Sparkes, M. Rancic, O. Pinel, M. Hosseini, P.K. Lam, B.C. Buchler, Phys. Rev. A 86, 023801 (2012). doi:10.1103/PhysRevA.86.023801

14. X.-W. Luo, J.J. Hope, B. Hillman, T.M. Stace, Phys. Rev. A 87, 062328 (2013). doi:10.1103/PhysRevA.87.062328

15. W. Franzen, Phys. Rev. 115, 850 (1959). doi:10.1103/PhysRev. 115.850

16. K.L. Corwin, Z.T. Lu, C.F. Hand, R.J. Epstein, C.E. Wieman, Appl. Opt. 37, 3295 (1998). doi:10.1364/AO.37.003295

17. T.T. Tsay, K.A. Jacobson, Biophys. J. 60, 360 (1991). doi:10. 1016/S0006-3495(91)82061-6

18. R. Chrapkiewicz, W. Wasilewski, C. Radzewicz, (2013). arXiv: 1304.5396

19. J. Vanier, J. Simard, J. Boulanger, Phys. Rev. A 9 (1974). doi:10. 1103/PhysRevA.9.1031

20. M. Arditi, T.R. Carver, Phys. Rev. 136, A643 (1964). doi:10. 1103/PhysRev.136.A643

21. F. Franz, Phys. Rev. 139, A603 (1965). doi:10.1103/PhysRev. 139.A603 
22. M.A. Bouchiat, J. Chem. Phys. 56, 3703 (1972). doi:10.1063/1. 1677750

23. E.L. Cussler, Diffusion: Mass Transfer in Fluid Systems. 3rd edn. (Cambridge University Press, Cambridge, 2009)

24. M.H. Ghatee, F. Niroomand-Hosseini, J. Chem. Phys. 126, 014302 (2007). doi:10.1063/1.2403879
25. K. Gibble, A. Gallagher, Phys. Rev. A 43, 1366 (1991). doi:10. 1103/PhysRevA.43.1366

26. M. Hosseini, B.M. Sparkes, G. Hétet, J.J. Longdell, P.K. Lam, B.C. Buchler, Nature 461, 241 (2009). doi:10.1038/nature08325

27. A. Fink, D. Baumer, E. Brunner, Phys. Rev. A 72, 053411 (2005). doi:10.1103/PhysRevA.72.053411 